\section{Runx2 inhibits chondrocyte proliferation and hypertrophy through its expression in the perichondrium}

\author{
Eiichi Hinoi, ${ }^{1,2,6}$ Peter Bialek, ${ }^{2,6}$ \\ You-Tzung Chen, ${ }^{3}$ Marie-Therese Rached, ${ }^{2}$ \\ Yoram Groner, ${ }^{4}$ Richard R. Behringer, ${ }^{3}$ \\ David M. Ornitz, ${ }^{5}$ and Gerard Karsenty ${ }^{1,2,7}$ \\ ${ }^{1}$ Department of Genetics and Development, Columbia \\ University, College of Physicians and Surgeons, New York, \\ New York 10032, USA; ${ }^{2}$ Department of Molecular \\ and Human Genetics, Baylor College of Medicine, Houston, \\ Texas 77030, USA; ${ }^{3}$ Department of Molecular Genetics, \\ University of Texas M.D. Anderson Cancer Center, Houston, \\ Texas 77030, USA; ${ }^{4}$ Department of Molecular Genetics, \\ Weizmann Institute of Science, Rehovot 76100, Israel; \\ ${ }^{5}$ Department of Molecular Biology and Pharmacology, \\ Washington University School of Medicine, \\ St. Louis, Missouri 63110, USA
}

The perichondrium, a structure made of undifferentiated mesenchymal cells surrounding growth plate cartilage, regulates chondrocyte maturation through poorly understood mechanisms. Analyses of loss- and gain-of-function models show that Twist-1, whose expression in cartilage is restricted to perichondrium, favors chondrocyte maturation in a Runx2-dependent manner. Runx2, in turn, enhances perichondrial expression of $F g f 18$, a regulator of chondrocyte maturation. Accordingly, compound heterozygous embryos for Runx2 and Fgf18 deletion display the same chondrocyte maturation phenotype as Fgf18-null embryos. This study identifies a transcriptional basis for the inhibition of chondrocyte maturation by perichondrium and reveals that Runx2 fulfills antagonistic functions during chondrogenesis.

Supplemental material is available at http://www.genesdev.org.

Received August 16, 2006; revised version accepted September 11, 2006.

Endochondral bone formation is a complex process that involves several cell types with distinct patterns of gene expression. The initial step in this process is characterized by the aggregation of undifferentiated mesenchymal cells expressing $\alpha 1(I)$ and $\alpha 1$ (III) Collagen into condensations at the location and with the overall shape of future skeletal elements. Subsequently, cells within these condensations differentiate into chondrocytes that do not express any more $\alpha 1$ (I) Collagen but instead, and among other genes, $\alpha 1(I I)_{b}$ Collagen. Cartilage anlagen

[Keywords: Twist-1; Runx2; FGF18; perichondrium; chondrocyte maturation]

${ }^{6}$ These authors contributed equally to this work.

${ }^{7}$ Corresponding author.

E-MAIL gk2172@columbia.edu; FAX (212) 923-2090.

Article published online ahead of print. Article and publication date are online at http://www.genesdev.org/cgi/doi/10.1101/gad.1482906. then enlarge through proliferation of chondrocytes that elongate to form prehypertrophic chondrocytes that will eventually exit the cell cycle to become bona fide hypertrophic chondrocytes. Genetically, hypertrophic chondrocytes differ from proliferating chondrocytes as they express $\alpha 1(X)$ Collagen but not $\alpha 1$ (II) Collagen (Kronenberg 2003). Throughout skeletogenesis, layers of $\alpha 1(I)$ Collagen-expressing undifferentiated mesenchymal cells persist and surround cartilage anlagen to form a structure called the perichondrium (Kronenberg 2003).

The transcriptional control of cell differentiation during skeletogenesis has been a topic of intense studies in the last $10 \mathrm{yr}$, leading to the identification of several key genes. For instance, Sox 9 along with Sox 5 and Sox 6 are seen as the main transcription factors triggering mesenchymal condensations and initiating chondrocyte differentiation (Lefebvre et al. 1998; Bi et al. 1999; Smits et al. 2001). On the other hand, Runx2 appears to be the earliest transcriptional determinant of osteoblast differentiation (Ducy et al. 1997; Komori et al. 1997; Otto et al. 1997). Runx2 has broader functions during skeletogenesis since it is, along with Runx3, an inducer of chondrocyte hypertrophy (Takeda et al. 2001; Ueta et al. 2001; Yoshida et al. 2004). This latter role of Runx 2 is explained by its transient expression in prehypertrophic chondrocytes.

Runx2 is also expressed at high levels and throughout skeletogenesis in cells of the perichondrium, suggesting that it may have additional roles during chondrogenesis (Ducy et al. 1997). This hypothesis is further supported by the recognized influence exerted by the perichondrium on chondrocyte maturation defined here as chondrocyte proliferation and hypertrophy. Indeed, removal of the perichondrium from chicken tibia organ cultures results in an increase in chondrocyte proliferation and in a larger zone of chondrocyte hypertrophy (Long and Linsenmayer 1998; Di Nino et al. 2001), implying that a yet unknown genetic cascade takes place in perichondrium to inhibit chondrocyte maturation.

While studying the regulation of Runx2 osteogenic function, we observed that the nuclear protein Twist-1 inhibits it by binding to its DNA-binding domain through a novel domain, the Twist box (Bialek et al. 2004). Part of this demonstration relied on the use of a hypomorphic Twist-1 allele called Charlie Chaplin (CC) $C C)$ in which a missense mutation in the Twist box decreases Twist-1's ability to interact with Runx2. We show here that chondrocyte maturation is regulated by Twist-1 although it is never expressed in chondrocytes during development. Instead, Twist-1 expression is restricted to cells of the perichondrium, where it regulates the function of Runx2 that in turn controls expression of Fgf18, a negative regulator of chondrocyte maturation (Liu et al. 2002; Ohbayashi et al. 2002). This study proposes a genetic and molecular basis for the role exerted by the perichondrium on chondrocyte maturation. In addition, it demonstrates that Runx2 exerts a broader array of functions during chondrogenesis than initially thought.

\section{Results and Discussion}

Twist-1 favors chondrocyte maturation

With the original purpose of analyzing Twist-1 functions during osteoblast differentiation, we generated trans- 
genic embryos expressing, under the control of $\alpha 1$ (I) Collagen regulatory elements that are active in mesenchymal cells (Rossert et al. 1995), an HA-tagged Twist-1 molecule or an HA-tagged truncated Twist-1 molecule containing Twist-1's N-terminal region (HA-Twist- $1_{N}$ ) (Fig. 1A). This latter form of Twist-1 contains its nuclear localization signal but does not affect Runx2 function (Bialek et al. 2004). These transgenic embryos were analyzed at embryonic day 15.5 (E15.5) and E16.5. Real-time PCR showed an $\sim 1.5$-fold increased expression of Twist-1 and Twist $-1_{N}$ in all founder transgenic embryos, and immunohistochemical study established that HA-Twist-1 and HA-Twist- $1_{\mathrm{N}}$ proteins were present in cells of the bone collar and perichondrium in ribs and humeri but not in chondrocytes of transgenic embryos (Fig. 1B,C; Supplementary Fig. 1A).

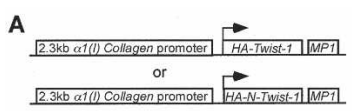

B

C

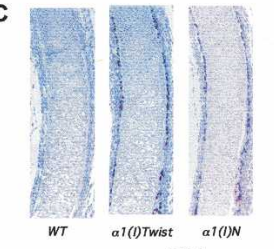

$\mathrm{E}$
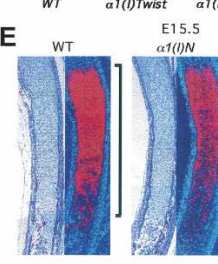

D

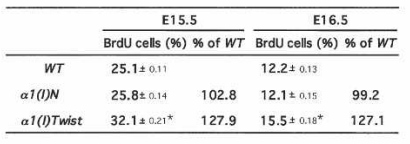

\begin{tabular}{lllll}
$\alpha 1(1)$ Twist $^{2}$ & $25.8 \pm 0.14$ & 102.8 & $12.1 \pm 0.15$ & 99.2 \\
\hline
\end{tabular}
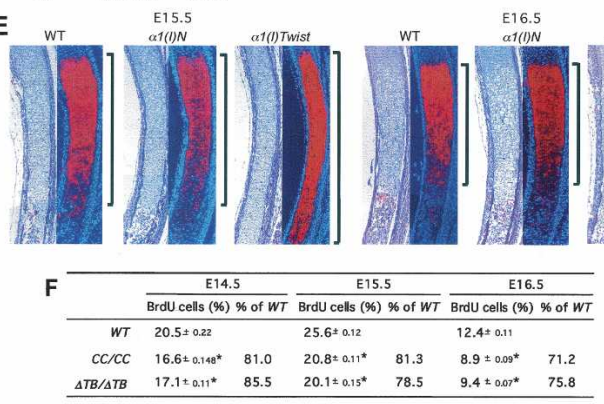

G
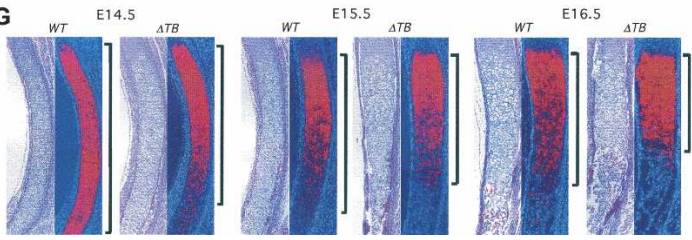

Figure 1. Twist-1 regulates chondrocyte maturation. (A) Schematic representation of the $\alpha 1(I)$ Collagen-Twist and $\alpha 1(I)$ Collagen-Twist $_{N}$ constructs. (B) Real-time PCR analysis for Twist-1 of wild-type (WT), $\alpha 1\left(\right.$ I) Collagen-Twist, and $\alpha 1\left(\right.$ I) Collagen-Twist ${ }_{N}$ expression. $(C)$ Immunohistochemical analysis of ribs of wild-type

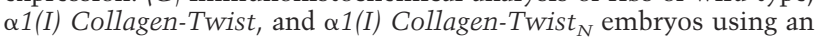
anti-HA antibody. Immunoreactivity against HA is seen in perichondrium of $\alpha 1$ (I) Collagen-Twist and $\alpha 1$ (I) Collagen-Twist ${ }_{N}$ skeletal elements. (D) BrdU incorporation analysis in ribs of E15.5 and E16.5 wild-type, $\alpha 1$ (I) Collagen-Twist, and $\alpha 1$ (I) Collagen-Twist ${ }_{N}$ embryos. Increased chondrocyte proliferation in $\alpha 1$ (I) CollagenTwist but not in $\alpha 1\left(\right.$ I) Collagen-Twist embryos. $_{N}(E)$ Histological analysis and in situ hybridization for $\alpha 1(X)$ Collagen expression in ribs of E15.5 and E16.5 wild-type, $\alpha 1(I)$ Collagen-Twist, and $\alpha 1$ (I)

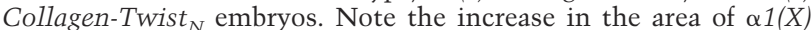
Collagen expression in $\alpha 1$ (I) Collagen-Twist embryos. (F) BrdU incorporation analysis in ribs of E14.5, E15.5, and E16.5 wild-type, $C C / C C$, and $\triangle T B^{-1-}$ embryos. Note the decrease in chondrocyte proliferation in $\mathrm{CC} / \mathrm{CC}$ and $\Delta \mathrm{T} B^{-1-}$ ribs. $(G)$ Histological analysis and in situ hybridization for $\alpha 1(X)$ Collagen expression in ribs of E14.5, E15.5, and E16.5 wild-type and $\Delta \mathrm{T}^{-/-}$embryos. Note the decreased expression of $\alpha 1(X)$ Collagen in $\Delta T B$ ribs.
Remarkably, E16.5 $\alpha 1$ (I) Collagen-HA-Twist-1 embryos displayed a marked advance in chondrocyte maturation in both ribs and humeri (Fig. 1D,E; Supplementary Fig. 1B,C). This was characterized by a significant increase in chondrocyte proliferation measured by BrdU incorporation and by an increase of the extent of the zone of hypertrophic chondrocytes determined histologically and by $\alpha 1(X)$ Collagen expression. In contrast, $\alpha 1(I)$ Collagen-HA-Twist- $1_{N}$ embryos did not show any abnormality in chondrocyte maturation (Fig. 1D,E; Supplementary Fig. 1B,C).

This observation was surprising since an overexpression of Twist-1 should result in a decrease in the activity of Runx2, a factor inducing chondrocyte hypertrophy (Takeda et al. 2001; Ueta et al. 2001). Thus, to determine if this was revealing an important biological function of Twist-1, we turned our attention to two loss-of-function models. The first one, the CC/CC mouse, harbors a missense mutation in the Twist box that decreases Twist-1's ability to interact with Runx2 and possibly other proteins. The second one, generated through homologous recombination in embryonic stem (ES) cells, lacks the entire Twist-box domain $\left(\Delta T B^{-1-}\right)$ (Supplementary Fig. $2)$. Both mutant mouse strains displayed major patterning defects affecting fore- and hindlimbs (data not shown); therefore we restricted our analysis of chondrocyte maturation to the ribs that are normally patterned in these mutant embryos. BrdU incorporation showed that chondrocyte proliferation was decreased in $C C / C C$ and $\Delta T B^{-/-}$embryos at all stages analyzed (Fig. 1F). Chondrocyte hypertrophy, whether it was analyzed histologically or through $\alpha 1(X)$ Collagen expression, was also less advanced in $\Delta T B^{-1-}$ compared with wild-type embryos (Fig. 1G). These results are the mirror image of what was observed in $\alpha 1$ (I) Collagen-Twist-1 embryos; thus, gain- and loss-of-function models concur to show that favoring chondrocyte maturation is a biological function of Twist-1.

\section{Twist-1 is not expressed and has no overt function in chondrocytes}

To elucidate how Twist-1 could regulate chondrocyte maturation, we first analyzed its pattern of expression between E13.5 and E16.5 in developing ribs and limbs. At both stages, Twist-1 expression could be detected in $\alpha 1$ (I) Collagen-expressing cells of the bone collar and perichondrium but not in $\alpha 1$ (II) Collagen-expressing proliferating chondrocytes or $\alpha 1(X)$ Collagen-expressing hypertrophic chondrocytes (Fig. 2A; Supplementary Fig. 3).

That in situ hybridization failed to detect Twist-1 expression in chondrocytes did not formally exclude that Twist-1 could be expressed in these cells below the limit of detection of in situ hybridization and yet affect chondrocyte maturation. To address this possibility, we deleted Twist-1 from nonhypertrophic chondrocytes by crossing mice harboring a floxed allele of Twist-1 (Twist$1^{\text {flox }}$ ) with $\alpha 1$ (II) Collagen-Cre transgenic mice to obtain Twist-1 $\mathrm{ch}^{-/-}$mice (Supplementary Fig. 4). The regulatory elements of the $\alpha 1$ (II) Collagen gene used to express Crerecombinase in chondrocytes are inactive in cells of the perichondrium (Takeda et al. 2001); thus, the $\alpha 1$ (II) Collagen-Cre transgenic mouse can delete genes of interest from nonhypertrophic chondrocytes but not from perichondrial cells. Histological examination of developing ribs and limbs did not show any overt difference in the 

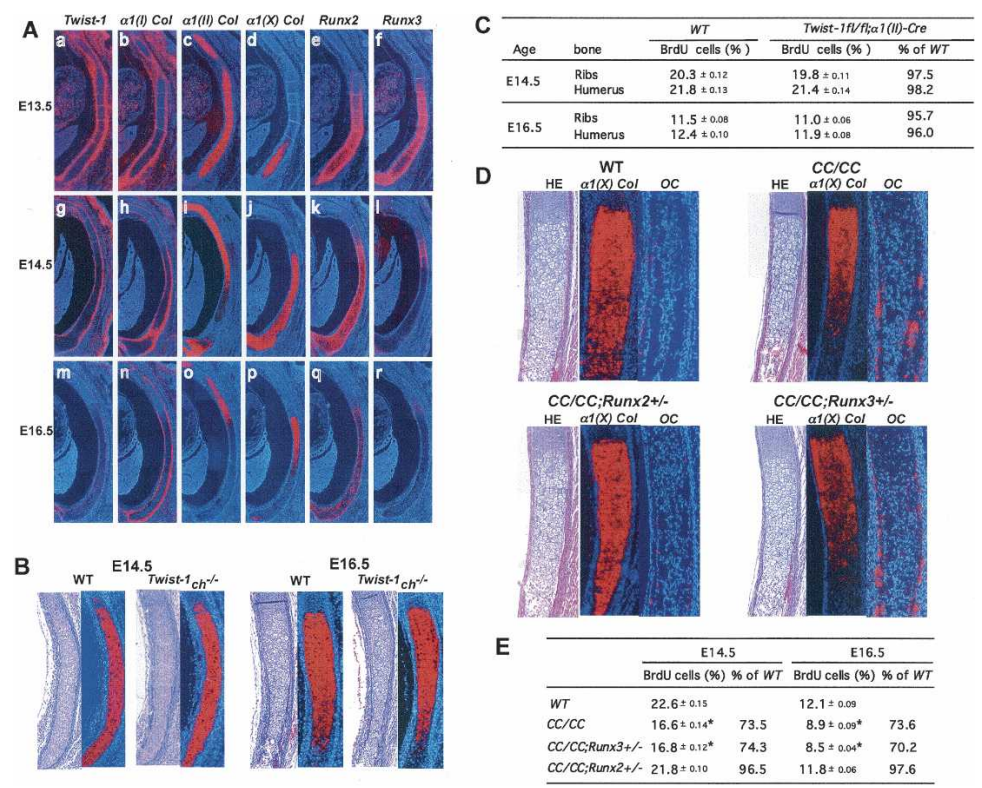

Figure 2. Twist-1 regulates Runx2 function in perichondrium. $(A)$ In situ hybridization for Twist-1 (panels $a, g, m$ ), $\alpha 1$ (I) Collagen (panels $b, h, n), \alpha 1$ (II) Collagen (panels $c, i, o$ ), $\alpha 1(X)$ Collagen (panels $d, i, p$ ), Runx2 (panels $e, k, q$ ), and Runx3 (panels $f, 1, r$ ) embryos at E13.5 (panels $a-f$ ), E14.5 (panels $g-1$ ), and E16.5 (panels $m-r$ ). Twist-1 is coexpressed with Runx2 in perichondrium and bone collar. $(B)$ Histological analysis and in situ hybridization for $\alpha 1(X)$ Collagen in ribs of E14.5 and E16.5 wild-type (WT) and $\alpha 1$ (II) Collagen-Cre; Twist- $1^{\text {flox/flox }}$ embryos. No change was observed in $\alpha 1(X)$ Collagen expression. $(C)$ BrdU incorporation analysis in ribs and humeri of E14.5 and E16.5 wild-type and $\alpha 1$ (II) Collagen-Cre; Twist-1 flox/flox embryos. No change in BrdU uptake was observed. $(D)$ Histological analysis and in situ hybridization for $\alpha 1(X)$ Collagen and Osteocalcin expression in ribs of E16.5 wild-type, CC/CC, CC/CC; Runx $2^{+/-}$, and CC/CC; Runx $^{+/-}$embryos. The accelerated chondrocyte hypertrophy observed

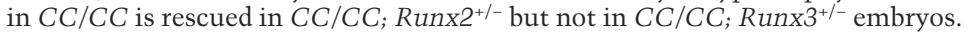
$(E)$ BrdU incorporation analysis in ribs of E14.5 and E16.5 wild-type, CC/CC,

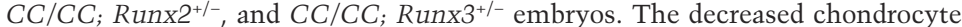

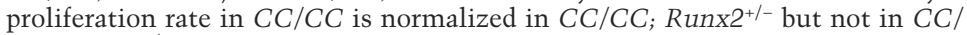
CC; Runx $3^{+/-}$embryos.

extent of the zone of proliferating chondrocytes or in the onset of chondrocyte hypertrophy between Twist-1 $\mathrm{ch}^{-1}$ and wild-type embryos at E14.5 and E16.5. This was verified molecularly through the analysis of $\alpha 1(X)$ Collagen expression and BrdU incorporation assays (Fig. 2B,C; Supplementary Fig. 4D). This absence of histological, cellular, or molecular abnormalities in mice harboring a chondrocyte-specific inactivation of Twist-1 is in agreement with Twist-1 expression studies and suggests that this gene influences chondrocyte proliferation through an indirect mechanism, presumably through its expression in perichondrial cells.

\section{Runx2 haploinsufficiency normalizes chondrocyte maturation in CC/CC embryos}

That the Twist box was required for Twist-1 regulation of chondrocyte maturation suggested that this process might occur through Twist-1 interaction with other nuclear proteins, possibly of the Runt family. To determine if this could be the case, we studied Runx2 and Runx3 expression in the perichondrium of developing ribs and limbs between E13.5 and E16.5. Runx2 was more strongly expressed in $\alpha 1$ (I) Collagen-expressing cells of the perichondrium, where Twist-1 is also expressed, than in $\alpha 1$ (II) Collagen-expressing chondrocytes throughout this period of development. In contrast, Runx3 expression in perichondrial cells faded away beyond E13.5 (Fig. 2A; Supplementary Fig. 3).

To determine whether Twist- 1 affects chondrocyte maturation through its interaction with Runx proteins in the perichondrium, we generated $C C / C C$ embryos lacking one copy of either Runx2 or Runx3 and analyzed chondrogenesis in these compound mutant embryos. Remarkably, removing one allele of Runx2 normalized chondrocyte proliferation measured by BrdU incorporation in E14.5 and E16.5 CC/CC ribs (Fig. 2E). Likewise, the extent of the hypertrophic chondrocyte zone, determined histologically and through $\alpha 1(X)$ Collagen expression, was similar in CC/ $C C$; Runx $2^{+/-}$and wild-type embryos, while it was reduced in CC/CC embryos (Fig. 2D). Accordingly, osteoblast differentiation defined by Osteocalcin expression was delayed in $C C / C C$; Runx $2^{+-}$and wild-type compared with $C C / C C$ embryos (Fig. 2D). In contrast, removing one allele of Runx3 did not affect any cellular, histological, and molecular events in CC/CC ribs (Fig. $2 \mathrm{D}, \mathrm{E})$. This is in agreement with the absence of Runx3 expression in perichondrium beyond E13.5. Taken together, these results provide genetic evidence suggesting that one mechanism whereby Twist- 1 regulates chondrocyte maturation is through its ability to inhibit Runx2 function in perichondrial cells.

\section{Fgf18 as a mediator of Runx2 inhibition of chondrocyte maturation}

To elucidate how Runx2, through its perichondrial expression, inhibits chondrocyte maturation, we focused on genes expressed in perichondrial cells, harboring putative Runx2-binding sites and encoding secreted factors regulating chondrocyte maturation in vivo. Fgf18 is one gene fulfilling all these criteria (Liu et al. 2002; Ohbayashi et al. 2002).

The study of Fgf18 expression in wild-type and various mouse mutant embryos with different levels of Runx2 activity supported the hypothesis that it may be a Runx2 target gene in perichondrial cells. Indeed, when compared with wild-type embryos, Fgf18 expression in perichondrial cells of ribs and humeri was higher in E15.5 and E16.5 CC/CC and $\Delta \mathrm{T} B^{-/-}$embryos, two mutant strains characterized by an increase in Runx2 activity, while it was decreased in Runx $2^{-/-}$and $\alpha 1(I)$ CollagenHA-Twist-1 embryos, which display a decrease in Runx2 activity (Fig. 3A; Bialek et al. 2004).

To provide a more compelling proof that Fgf18 and Runx2 interact in perichondrial cells to control chondrocyte maturation, we compared embryos heterozygous for both Runx2 and Fgf18 inactivation to Fgf18 ${ }^{-/-}$embryos. Chondrocyte proliferation was nearly identical in E14.5 and E16.5 wild-type, Runx2 $2^{+/}$, and $F g f 18^{+/-}$embryos. In

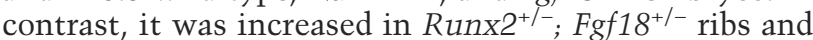
humeri to the same extent as in $F g f 18^{-/-}$ribs and humeri

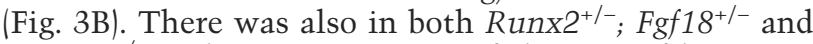
in $\mathrm{Fg}_{18^{-/}}$embryos an increase of the area of hypertrophic chondrocytes determined by $\alpha 1(X)$ Collagen expression and a delay in osteoblast differentiation, defined by Osteocalcin expression (Fig. 3C). 
A
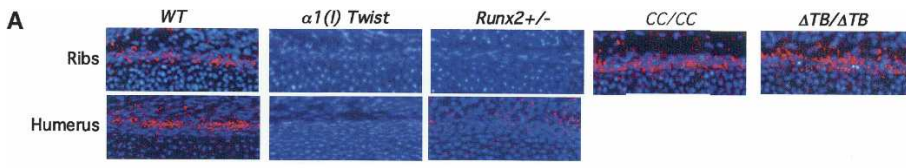

B

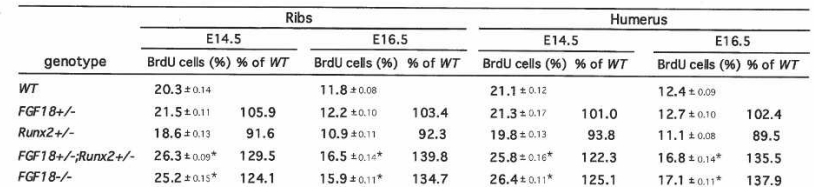

C
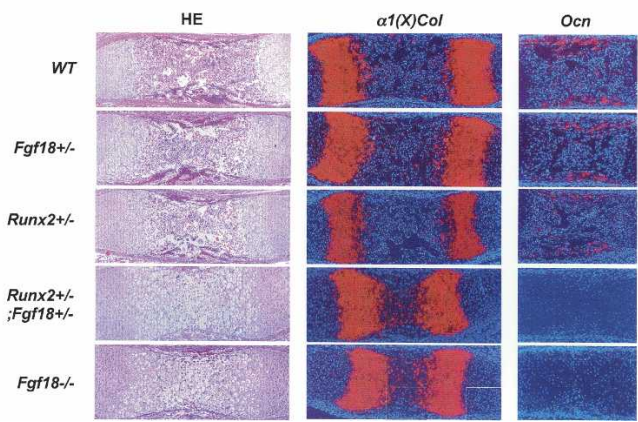

D

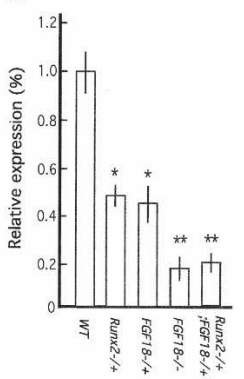

Figure 3. Runx2 regulates chondrocyte proliferation through Fgf18. (A) In situ hybridization analysis of Fgf18 expression in ribs and humeri of E15.5 wild-type (WT), $\alpha 1(I)$ Twist-1, Runx $2^{+/-}, C C / C C$, and $\Delta$ Twist box ${ }^{-/-}$embryos. Perichondrial Fgf18 expression is decreased in $\alpha 1$ (I) Twist 1 and Runx $2^{+/-}$and increased in $C C / C C$ and $\Delta$ Twist box ${ }^{-/-}$embryos. $(B)$ BrdU incorporation analysis in ribs and humeri at E14.5 and E16.5 of wild-type, Fgf18 ${ }^{+/-}$, Runx2 $^{+/-}, \mathrm{Fgfl}^{+8^{+-}}$; Runx2 $2^{+/-}$, and $\mathrm{Fgf18^{-/- }}$ embryos. Chondrocyte proliferation is similarly increased in $\mathrm{Fgf18^{+- } -}$;

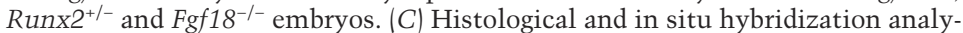
sis of $\alpha 1(X)$ Collagen and Osteocalcin expression in humeri of E16.5 wild-type,

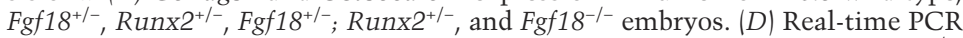
analysis of $\alpha 1$ Integrin expression in humeri of E15.5 wild-type, Runx2 $2^{+-}$,

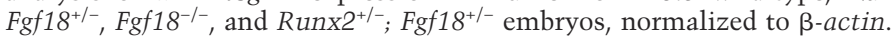

To verify molecularly the existence of an interaction between Runx2 and Fgf18, we analyzed through realtime PCR expression of $\alpha 1$ integrin, a target gene of Fgf18 expressed in proliferating chondrocytes (Supplementary Fig. 5; Davidson et al. 2005). Expression of $\alpha 1$ integrin was reduced $80 \%$ in $F g f 18^{--}$compared with wild-type humeri (Fig. 3D). The same was true in humeri of het-

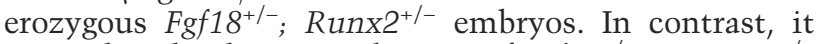
was reduced only $40 \%$ in humeri of $F g f 18^{+/-}$or Runx $2^{+/-}$ embryos (Fig. 3D). Taken together, these studies establish the existence of a genetic interaction between Fgf18 and Runx2 taking place in perichondrial cells and resulting in a delay in chondrocyte maturation.

\section{Regulation of Fgf18 expression by Runx2}

To establish molecularly that Fgf18 is a transcriptional direct target of Runx2, we analyzed the Fgf18 locus and found two potential Runx2-binding sites in the promoter region (sites 1 and 2) (Fig. 4A). The relevance of these sites was first tested by electric mobility shift assay (EMSA) using wild-type osteoblast nuclear extracts (NEs) and, as probes, labeled double-stranded oligonucleotides containing sequences covering them. A protein-DNA complex formed upon incubation of site 1 or of site 2 with osteoblast NEs (Fig. 4B). Moreover, an antibody against Runx2 supershifted these protein-DNA complexes, while antibodies against Spl did not. Second, to verify that Runx2 binds to the two sites present in the Fgf18 promoter in vivo, we performed chromatin immu- noprecipitation analyses using primary osteoblasts. Antibodies against Runx2 immunoprecipitated the regions of the Fgf18 promoter containing either site 1 or site 2 , while they could not immunoprecipitate Fgf18 coding sequences.

To assess the importance of sites 1 and 2 in determining Fgf18 promoter activity, we performed DNA transfection experiments in ROS17/2.8 osteoblastic cells that express Runx2 (Ducy and Karsenty 1995). We used as a reporter construct a vector containing a 1.6-kb fragment of the Fgf18 promoter fused to the luciferase gene (pFgf18-Luc) and as a positive control the 147-bp fragment of the Osteocalcin gene2 promoter fused to the luciferase gene (pOG2-luc). A mutation in either site 1 or site 2 of the Fgf 18 promoter caused a $40 \%$ decrease of pFgf18-Luc activity, while a mutation in both sites decreased it $60 \%$ (Fig. 4D). This result suggested that, in the conditions of this assay, the two Runx2-binding sites played an equally important role in determining activity of this fragment of Fgf18 promoter in cells of the osteoblast lineage, although other transcription factors must regulate Fgf18 promoter activity since mutation of these two sites decreased activity of this promoter only twofold. To further ascertain the importance of Runx2 as a regulator of Fgf18 promoter activity, we next used COS cells that do not express Runx2 or Fgf18 to perform DNA cotransfection experiments. In this assay, Runx2 transactivated equally well pFgf18-Luc or pOG2-luc. Moreover, mutations in either site 1 or site 2 in the Fgf18 promoter decreased Runx2 ability to transactivate $p F g f 18-L u c$ threefold, while mutations in both sites abolished it (Fig. 4E). Taken together, these data are consistent with the hypothesis that Fgf18 is a transcriptional target of Runx2.

Various experimental evidence gathered mostly in chick has convincingly shown that the poorly differentiated mesenchymal cells making up the perichondrium influence negatively chondrocyte maturation (Long and Linsenmayer 1998; Di Nino et al. 2001). In an effort to provide a molecular basis for this function of perichondrium, we followed the unexpected observation that Twist-1, a gene expressed in perichondrial cells but not in chondrocytes, favors chondrocyte maturation. That this function of Twist-1 occurred through the Twist box along with Runx2 expression in the perichondrium led us to show through several loss-of-function experiments that Runx2 regulates Fgf18 expression in perichondrium and thereby inhibits chondrocyte maturation. Since Runx2 expression is limited to part of the perichondrium, it is likely that other transcription factors may be involved in the regulation of chondrocyte maturation by the perichondrium. Likewise, we cannot rule out that Runx2 may have other targets in perichondrial cells that could explain the role of perichondrium on chondrocyte maturation.

Although no tools are available yet to delete genes in perichondrial cells only, several lines of evidence suggest that the Twist-1-Runx2-Fgf18 cascade described here takes place primarily in the perichondrium. First, it was identified through the analysis of mutants in Twist-1, a gene that is neither expressed nor active in proliferating chondrocytes; second, Twist-1 and Runx2 that are both 
A
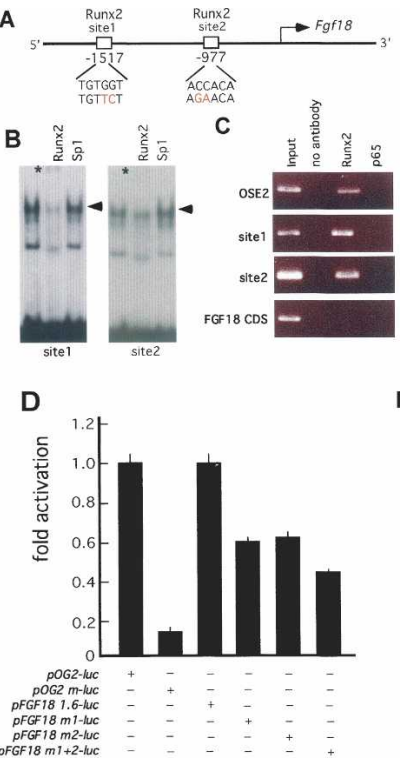

E
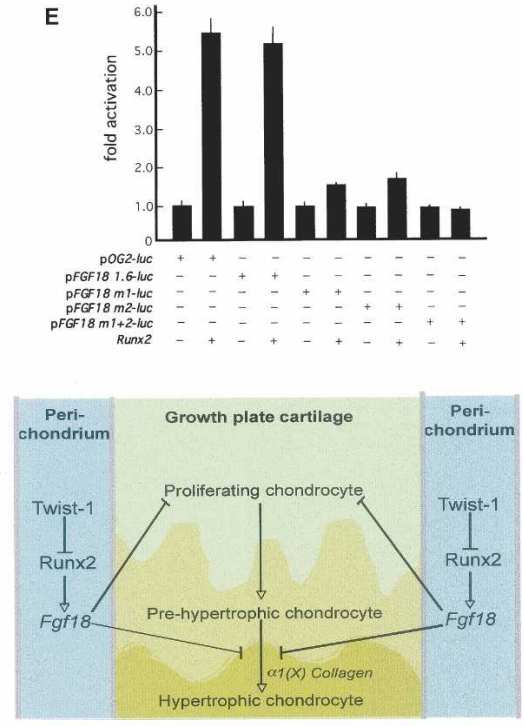

Figure 4. Fgf18 expression is regulated by Runx2. (A) Schematic representation of putative Runx2-binding sites in the promoter of Fgf18. (B) EMSA. Osteoblast NEs were incubated with labeled oligonucleotides encompassing Fgf18 sites 1 and 2. A protein-DNA complex formed upon incubation of NEs with all sites examined. Osteoblast NEs were incubated with antibodies against Runx2 or Sp1 prior to incubation with labeled oligonucleotides. Only antibody against Runx2 decreased binding to the all Fgf18 site (1 and 2) oligonucleotides. (C) ChIP assay. An antibody against Runx2 immunoprecipitated site 1 and site 2 of the Fgf18 promoter and the Osteocalcin promoter (OSE) but not Fgf18 coding sequence (CDS). In another negative control experiment, an antibody against the P65 component of NF-кB did not immunoprecipitate these two sites. $(D)$ ROS17/2.8 cells were transiently transfected with wild-type 1.6-kb Fgf18 promoter-luc (pFgf18 1.6-luc), Fgf18 promoter-luc, or pOG2-luc constructs with either site 1 or site 2, or both sites were mutated (pFgf18 m1-luc, pFgf18 m2-luc, or pFgf18 $\mathrm{m} 1+2$-luc, respectively). (E) COS7 cells were transiently transfected with either pFgf18 1.6-luc, pFgf18 m1-luc, pFgf18 m2-luc, pFgf18 m1 + 2-luc, or pOG2-luc constructs with or without Runx2 expression vector. Runx 2 increased activity of pFgf18 1.6-luc, but not of pFgf18 m1 + 2-luc. $(F)$ Proposed model. Twist-1 regulates Runx2 activity in perichondrial cells where Fgf18 is a target gene of Runx2. As a result, through its perichondrial expression, Runx2 exerts a negative influence on chondrocyte maturation.

expressed in perichondrial cells interact in this process; third, Fgf18 expression can only be detected in the perichondrium. We should emphasize that this genetic cascade may not be limited to the perichondrium, and it could also explain the craniosynostosis of Twist ${ }^{+/-}$mice since Fgf18 is known to regulate suture closure in the skull (Liu et al. 2002; Ohbayashi et al. 2002). In addition, the absence of osteocalcin expression observed in Runx $2^{+/-} ; \mathrm{Fg} f 18^{+/-}$embryos suggests that Runx2 may exert through Fgf18 a negative influence on osteoblast differentiation; this would be consistent with the increase in osteoblast differentiation observed in $\mathrm{Fg}_{\mathrm{f}} 18^{-/-} \mathrm{em}$ bryos and implies that Runx2 exerts positive and negative influences on bone formation (Liu et al. 2002; Ohbayashi et al. 2002).

Based on this and previous studies, it appears that Runx2 has more complex functions during chondrogenesis than initially thought (Fig. 4F). First, early during skeletogenesis and through its expression in prehypertrophic chondrocytes, Runx2 triggers chondrocyte hypertrophy in part by up-regulating $\alpha 1(X)$ Collagen expression (Takeda et al. 2001; Ueta et al. 2001; Zheng et al. 2003). Subsequently, through its longer lasting perichondrial expression, Runx2 exerts a negative influence on chondrocyte proliferation and hypertrophy. These antagonistic functions of Runx2 during chondrogenesis may help regulate bone formation. Although further experiments will be needed to test the validity of this model, results presented in this and previous studies illustrate the dynamic and complex influences that Runx 2 exerts on multiple cell differentiation events during skeletogenesis. In that respect, Runx2 and the Sox proteins appear to be the main transcriptional architects of skeletogenesis.

\section{Materials and methods}

Mutant animals

CC/CC, Runx2-, Runx3-, and Fgf18-deficient mice, and $\alpha 1$ (I) Collagen-Cre and $\alpha 1$ (II) Collagen-Cre transgenic mice were described previously (Ducy et al. 1997; Dacquin et al. 2002; Li et al. 2002; Liu et al. 2002; Bialek et al. 2004). $\alpha$ 1(I) Collagen-Twist and $\mathrm{N}$-Twist transgenic mice were generated using standard procedures. Genotyping was performed by PCR analysis of genomic DNA.

\section{In situ hybridization, immunohistochemistry, and BrdU} incorporation assay

In situ hybridization was performed using ${ }^{35} \mathrm{~S}$-labeled riboprobes. Hybridizations were performed at $60^{\circ} \mathrm{C}$. Autoradiography and Hoechst 33258 staining were performed as described (Takeda et al. 2001). HA-Twist-1 was detected with a mouse anti-HA antibody (1:1000; Roche) using the ABC Elite Kit (Vector Laboratories) and NovaRed (Vector Laboratories) as a substrate. For the proliferation assay, pregnant mice were injected with $500 \mu \mathrm{L}$ of $10 \mathrm{mM}$ BrdU $1 \mathrm{~h}$ before sacrifice. BrdU incorporation was detected using a Zymed BrdU staining kit. Sections were counterstained with hematoxylin. At least six embryos of each genotype were analyzed for each group and for each figure. Statistical significance was assessed by the Student's test.

RNA analysis and DNA transfection experiments

Total RNA was isolated from the indicated source, and realtime PCR was performed on an ABI Prism 7000 Sequence Detection System (Applied Biosystems). COS and ROS 17/2.8 cells were grown in DMEM and DMEM-F-12, respectively, with $10 \%$ FBS. The 1.6-kb fragment of the FGF18 promoter was PCRamplified from genomic DNA, and its sequence was verified and cloned into pGL3-luc. Mutagenesis was performed using a mutagenesis kit (Stratagene) as per the manufacturer's instructions. Results are expressed as fold induction compared with single transfection of each pair. An asterisk indicate a $P$ value $<0.05$; a double asterisk indicates a $P$ value $<0.01$.

DNA-binding assay and chromatin immunoprecipitation (ChIP) assay Labeled double-stranded oligonucleotides were prepared and EMSA was performed as described (Ducy and Karsenty 1995). Antibodies were added to the EMSA reaction and incubated on ice for $30 \mathrm{~min}$ prior to loading onto a $5 \%$ acrylamide gel. The ChIP assay was performed as per the manufacturer's instructions (Upstate Biotechnology) using primary osteoblasts. PCR primer sequences are available upon request. Primary osteoblasts were prepared and cultured as described (Ducy and Karsenty 1995).

\section{Acknowledgments}

We thank Dr. H. Kronenberg for providing training to P.B., Dr. P. Ducy for suggestions and critical reading of the manuscript, and M. Schrock and J. Bartlett for excellent technical assistance. This work was supported by the National Institutes of Health (G.K.), Japan Society for the Promotion of Science (E.H.), and the Arthritis Foundation (P.B.).

\section{References}

Bi, W., Deng, J.M., Zhang, Z., Behringer, R.R., and de Crombrugghe, B. 1999. Sox9 is required for cartilage formation. Nat. Genet. 22: 85-89. 


\section{Hinoi et al.}

Bialek, P., Kern, B., Yang, X., Schrock, M., Sosic, D., Hong, N., Wu, H., Yu, K., Ornitz, D.M., Olson, E.N., et al. 2004. A twist code determines the onset of osteoblast differentiation. Dev. Cell 6: 423-435.

Dacquin, R., Starbuck, M., Schinke, T., and Karsenty, G. 2002. Mouse $\alpha 1(\mathrm{I})$-collagen promoter is the best known promoter to drive efficient Cre recombinase expression in osteoblast. Dev. Dyn. 224: 245-251.

Davidson, D., Blanc, A., Filion, D., Wang, H., Plut, P., Pfeffer, G., Buschmann, M.D., and Henderson, J.E. 2005. Fibroblast growth factor (FGF) 18 signals through FGF receptor 3 to promote chondrogenesis. J. Biol. Chem. 280: 20509-20515.

Di Nino, D.L., Long, F., and Linsenmayer, T.F. 2001. Regulation of endochondral cartilage growth in the developing avian limb: Cooperative involvement of perichondrium and periosteum. Dev. Biol. 240: 433-442.

Ducy, P. and Karsenty, G. 1995. Two distinct osteoblast-specific cisacting elements control expression of a mouse osteocalcin gene. Mol. Cell. Biol. 15: 1858-1869.

Ducy, P., Zhang, R., Geoffroy, V., Ridall, A.L., and Karsenty, G. 1997. Osf2/Cbfa1: A transcriptional activator of osteoblast differentiation. Cell 89: 747-754.

Komori, T., Yagi, H., Nomura, S., Yamaguchi, A., Sasaki, K., Deguchi, K., Shimizu, Y., Bronson, R.T., Gao, Y.H., Inada, M., et al. 1997. Targeted disruption of cbfal results in a complete lack of bone formation owing to maturational arrest of osteoblasts. Cell 89: 755-764.

Kronenberg, H.M. 2003. Developmental regulation of the growth plate. Nature 423: 332-336.

Lefebvre, V., Li, P., and de Crombrugghe, B. 1998. A new long form of Sox5 (L-Sox5), Sox6 and Sox9 are coexpressed in chondrogenesis and cooperatively activate the type II collagen gene. EMBO I. 17: 57185733.

Li, Q.L., Ito, K., Sakakura, C., Fukamachi, H., Inoue, K., Chi, X.Z., Lee, K.Y., Nomura, S., Lee, C.W., Han, S.B., et al. 2002. Causal relationship between the loss of RUNX3 expression and gastric cancer. Cell 109: 113-124.

Liu, Z., Xu, J., Colvin, J.S., and Omitz, D.M. 2002. Coordination of chondrogenesis and osteogenesis by fibroblast growth factor 18. Genes \& Dev. 16: 859-869.

Long, F. and Linsenmayer, T.F. 1998. Regulation of growth region cartilage proliferation and differentiation by perichondrium. Development 125: 1067-1073.

Ohbayashi, N., Shibayama, M., Kurotaki, Y., Imanishi, M., Fujimori, T. Itoh, N., and Takada, S. 2002. FGF18 is required for normal cell pro liferation and differentiation during osteogenesis and chondrogenesis. Genes \& Dev. 16: 870-879.

Otto, F., Thornell, A.P., Crompton, T., Denzel, A., Gilmour, K.C., Rosewell, I.R., Stamp, G.W., Beddington, R.S., Mundlos, S., Olsen, B.R., et al. 1997. Cbfa1, a candidate gene for cleidocranial dysplasia syndrome, is essential for osteoblast differentiation and bone development. Cell 89: 765-771.

Rossert, J., Eberspaecher, H., and de Crombrugghe, B. 1995. Separate cis-acting DNA elements of the mouse pro- $\alpha 1(\mathrm{I})$ collagen promoter direct expression of reporter genes to different type I collagen-producing cells in transgenic mice. J. Cell Biol. 129: 1421-1432.

Smits, P., Li, P., Mandel, J., Zhang, Z., Deng, J.M., Behringer, R.R., de Crombrugghe, B., and Lefebvre, V. 2001. The transcription factors L-Sox 5 and Sox6 are essential for cartilage formation. Dev. Cell 1: 277-290.

Takeda, S., Bonnamy, J.P., Owen, M.J., Ducy, P., and Karsenty, G. 2001. Continuous expression of Cbfal in nonhypertrophic chondrocytes uncovers its ability to induce hypertrophic chondrocyte differentiation and partially rescues Cbfal-deficient mice. Genes \& Dev. 15: $467-481$.

Ueta, C., Iwamoto, M., Kanatani, N., Yoshida, C., Liu, Y., EnomotoIwamoto, M., Ohmori, T., Enomoto, H., Nakata, K., Takada, K., et al. 2001. Skeletal malformations caused by overexpression of Cbfal or its dominant negative form in chondrocytes. J. Cell Biol. 153: 87-100.

Yoshida, C.A., Yamamoto, H., Fujita, T., Furuichi, T., Ito, K., Inoue, K., Yamana, K., Zanma, A., Takada, K., Ito, Y., et al. 2004. Runx2 and Runx3 are essential for chondrocyte maturation, and Runx2 regulates limb growth through induction of Indian hedgehog. Genes \& Dev. 18: 952-963

Zheng, Q., Zhou, G., Morello, R., Chen, Y., Garcia-Rojas, X., and Lee, B. 2003. Type X collagen gene regulation by Runx 2 contributes directly to its hypertrophic chondrocyte-specific expression in vivo. J. Cell Biol. 162: 833-842. 


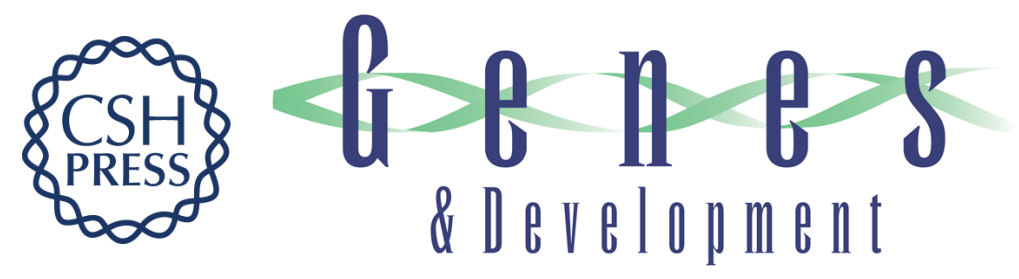

\title{
Runx2 inhibits chondrocyte proliferation and hypertrophy through its expression in the perichondrium
}

\author{
Eiichi Hinoi, Peter Bialek, You-Tzung Chen, et al.
}

Genes Dev. 2006, 20: originally published online October 18, 2006

Access the most recent version at doi:10.1101/gad.1482906

\section{Supplemental http://genesdev.cshlp.org/content/suppl/2006/10/17/gad.1482906.DC1 \\ Material}

References This article cites 21 articles, 11 of which can be accessed free at:

http://genesdev.cshlp.org/content/20/21/2937.full.html\#ref-list-1

\section{License}

Email Alerting

Receive free email alerts when new articles cite this article - sign up in the box at the top

Service

right corner of the article or click here.

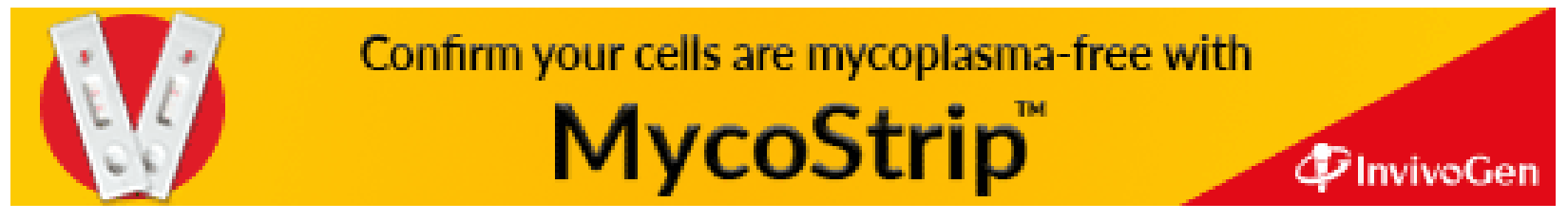

